\title{
CHARACTERIZING PROPERTIES FOR INCONSISTENCY INDICES IN THE AHP
}

\author{
Matteo Brunelli \\ IAMSR and Turku Centre for Computer Science \\ Åbo Akademi University, Joukahainengatan 3-5A, FIN-20520 Åbo, Finland \\ E-mailmatteo.brunelli@abo.fi \\ Michele Fedrizzi* \\ Department of Computer and Management Sciences, University of Trento \\ Via Inama 5, I-38122 Trento, Italy \\ E-mail michele.fedrizzi@unitn.it
}

\begin{abstract}
Several indices have been proposed in literature to evaluate the inconsistency of pairwise comparison matrices in the Analytic Hierarchy Process. Nevertheless, no commonly accepted definition of 'inconsistency index' exists. In this study present and discuss some characterizing properties for inconsistency indices in order to contribute to a formalization of this issue.
\end{abstract}

Keywords: analytic hierarchy process, pairwise comparison matrices, consistency indices, characterizing properties.

\section{Introduction}

Given a set of alternatives $X=\left\{x_{1}, \ldots, x_{n}\right\} \quad(n \geq 2)$, a pairwise comparison matrix $\mathbf{A}=\left[a_{i j}\right]$ is a $n \times n$ reciprocal positive matrix, $a_{i j}>0, a_{i i}=1, a_{i j} a_{j i}=1 \forall i, j$, where $a_{i j}$ is a subjective estimation on a ratio scale of the relative dominance of $x_{i}$ over $x_{j}$ (Saaty, 1977). A pairwise comparison matrix is defined to be consistent if and only if the transitivity condition

$$
a_{i k}=a_{i j} a_{j k} \quad \forall i, j, k
$$

holds. If $\mathbf{A}$ is consistent, then there exists a priority vector $\mathbf{w}=\left(w_{1}, \ldots, w_{n}\right)$ such that

$$
a_{i j}=\frac{w_{i}}{w_{j}} \quad \forall i, j .
$$

The reliability of the priority vector is related with the consistency of the pairwise judgments expressed by the decision maker and collected in the pairwise comparison matrix. In fact, the more consistent the judgments are, the more likely it is that the decision maker is a good expert with a deep insight into the problem and pays the due attention in eliciting his/her preferences. Conversely, if the judgments are far from consistency, it is likely that the expert expressed them with scarce competence and care. Therefore, in several applications it is crucial that the consistency evaluation process is carried out in a fair way.

So far, many methods have been proposed in literature in order to estimate inconsistency and we shortly present some of them, although we skip technical details. The first index to be introduced was the Consistency Index (Saaty, 1977),

\footnotetext{
* Corresponding author
} 


$$
C I(\mathbf{A})=\frac{\lambda_{\max }-n}{n-1}
$$

where $\lambda_{\max }$ is the principal right eigenvalue of $\mathbf{A}$. Saaty proposed also a more suitable measure of inconsistency, the Consistency Ratio $C R$,

$$
C R(\mathbf{A})=\frac{C I(\mathbf{A})}{R I} .
$$

The Random Index, $R I$, is defined as the average $C I$ over all the pairwise comparis on matrices of the same order of A. Golden and Wang proposed a method to compute the deviations between the entries of a pairwise comparison matrix and their theoretical values $w_{i} / w_{j}$ (Golden and Wang, 1989). Crawford and Williams, and lately Aguaròn and Moreno-Jimènez, proposed and refined an index that also computes distances between the decision maker's judgements and the ir theoretical values (Crawford and Williams, 1985) (Aguaron and Moreno-Jimènez, 2003). Barzilai formulated a normalized index based on squared errors (Barzilai, 1998). By using open unbounded scales, he was able to state several interesting mathematical properties. Pelàez and Lamata defined their inconsistency index for a pairwise comparison matrix as the average of all the determinants of its $3 \times 3$ submatrices, each containing a different transitivity of the original matrix (Pelaez and Lamata, 2003). Shiraishi, Obata, and Daigo proposed to use the third coefficient, $c_{3}$, of the characteristic polynomial of $\mathbf{A}$ as an index of inconsistency (Shiraishi, Obata, and Daigo 1998). Stein and Mizzi considered the general result that the columns of a consistent pairwise comparison matrix are proportional if and only if $\mathbf{A}$ is consistent. Thus, they formulated an index which takes into account how far the columns are from being proportional to each other (Stein and Mizzi, 2007). Koczkodaj and Duszak introduced a max-min based inconsistency index (Koczkodaj, 1993), (Duszak and Koczkodaj, 1994). Cavallo and D'Apuzzo characterized pairwise comparison matrices by means of Abelian linearly ordered groups and stated their inconsistency index (Cavallo and D'Apuzzo, 2009, 2010). Ramik, Korviny and Perzina proposed a normalized index based on some new metrics for fuzzy pairwise comparison matrices, but the index can be easily reported to a simpler form in order to deal with pairwise comparison matrices (Ramik and Korviny, 2010) (Ramik and Perzina, 2010).

The importance of having reliable inconsistency indices becomes evident by considering the various applications proposed in the literature where inconsistency evaluation plays a crucial role. Inconsistency indices have been employed to estimate missing entries and to improve the consistency of incomplete pairw ise comparison matrices (Lamata and Pelaez, 2002) (Obata, Shiraishi, Daigo and Nakajima, 1999) (Xu and Cuiping, 1999). They have been also used in group decision making in order to give different weights to experts ac cording to the ir judgements' consistency.

This paper originates from the remark that many different definitions of inconsistency indices have been proposed, but the basic questions: 'what is a consistency index?', 'which are the minimal characterizing properties that a proposed index must satisfy in order to be considered an inconsistency index?' have never been addressed. The aim of this paper is to introduce and justify some characterizing properties for inconsistency indices. Section 2 introduces some necessary notation and four characterizing properties. Section 3 presents conclusions and ideas for future research.

\section{Char acterizing properties}

For notational convenience, we denote by $A$ the set of all the $n \times n$ pairwise comparison matrices, i.e. positive reciprocal matrices of order $n$. Similarly, we denote by $A *$ the set of all the $n \times n$ consistent pairwise comparison matrices. By the simplest approach, a matrix in $A$ can therefore either be consistent or non-consistent (inconsistent). More generally, we assume that an inconsistency index is a function $I: A \rightarrow \square$ satisfying a set of properties described in the following. We argue the necessity of these properties for $I(\mathbf{A})$ to provide a satisfactory measure of how much $\mathbf{A}$ deviates from 
full consistency condition (1). The first two properties are simple regularity conditions, while the second and the third are more specific and characterizing.

\subsection{Property 1. Existence of a unique e le ment representing cons is tency}

The first characterizing property requires that a unique real number must represent perfect consistency. More formally, it is demanded that

$$
\exists ! v \in \square \text { such that } I(\mathbf{A})=v \Leftrightarrow \mathbf{A} \in A^{*} .
$$

In the case of Saaty's index (3), for example, it is $v=0$,

$$
\mathrm{CI}(\mathbf{A})=0 \Leftrightarrow \mathbf{A} \in A^{*} .
$$

Without loss of generality, we can assume that

$$
I(\mathbf{A})>v \Leftrightarrow \mathbf{A} \in A-A^{*} .
$$

\subsection{Property 2. Invariance unde $r$ pe rmutation of alte rnatives}

This second property is similar to the axiom of anonymity of social choice and requires that an inconsistency index should be independent from the order in which the alternatives are presented. Formally,

$$
I(\mathbf{A})=I\left(\mathbf{P A P}^{\mathrm{T}}\right) \quad \forall \mathbf{A} \in A
$$

Where $\mathbf{P}$ is any $n \times n$ permutation matrix, i.e. a square binary matrix with exactly one entry equal to 1 on each row and each column and 0's elsewhere.

\subsection{Property 3. Weak monotonicity under reciprocity preserving mapping}

Before formalizing the third property, we try to describe its meaning. The underlying idea is that if inconsistency is emphasized, then an inconsistency index cannot return a better value. We consider that inconsistency is emphasized when the decision maker's preferences are intensified. As a starting point, let we consider the case with full indifference between alternatives, $a_{i j}=1 \quad \forall i j$. These preferences are trivially consistent. Going farther from this uniformity means having stronger judgments and this should make more evident their possible inconsistency. In other words, intensifying the preferences (pushing them away from indifference) should emphasize the characteristics of these preferences and their possible contradictions. Thus, the condition at issue requires that, if preferences are intensified, then the inconsistency index cannot diminish. Clearly the crucial point is to find a transformation which can intensify preferences and preserve their structure at the same time. In the follow ing, we are going to prove that such a transformation exists and is unique.

Given $\mathbf{A}=\left[a_{i j}\right] \in A$, we denote by $f$ such transformation, $\hat{a}_{i j}=f\left(a_{i j}\right) i, j=1, \ldots, n$. The newly constructed matrix $\hat{\mathbf{A}}=\left[\hat{a}_{i j}\right]$ obtained from $\mathbf{A}$ by means of $f$ must be positive and reciprocal, that is it still must belong to $A$. Hence

and then

$$
\hat{a}_{j i}=1 / \hat{a}_{i j},
$$

$$
\begin{gathered}
f\left(a_{j i}\right)=1 / f\left(a_{i j}\right) \\
f\left(1 / a_{i j}\right)=1 / f\left(a_{i j}\right)
\end{gathered}
$$




$$
f\left(a_{i j}\right) f\left(1 / a_{i j}\right)=1,
$$

or, more compactly, with $a_{i j}=x$,

$$
f(x) f(1 / x)=1 .
$$

Equation (7) is a special case, for $y=1 / x$, of the well known Cauchy functional equation

$$
f(x) f(y)=f(x y) .
$$

Note that $f(x(1 / x))=f(1)=1$ since function $f$ must map diagonal elements 1 into the same value 1 in order to respect $\hat{\mathbf{A}} \in \boldsymbol{A}$. Taking into account positivity of arguments, $x=a_{i j}>0$, it is therefore sufficient to assume continuity of $f$ in order to have the following unique solution of (8) and consequently of (7) (Aczel, 1966),

$$
f(x)=x^{b}, \quad b \in \square .
$$

Therefore, the only continuous transformation $f\left(a_{i j}\right)$ preserving reciproc ity is (9), i.e.

$$
f\left(a_{i j}\right)=a_{i j}^{b} \text {. }
$$

Clearly, for $b>1$ each entry $a_{i j} \neq 1$ is moved away from indifference value 1 , thus representing an intensification of the preferences:

$$
\begin{aligned}
& a_{i j}>1 \Rightarrow a_{i j}^{b}>a_{i j}>1 \\
& 0<a_{i j}<1 \Rightarrow 0<a_{i j}^{b}<a_{i j}<1
\end{aligned}
$$

The opposite occurs for $0<b<1$, thus representing a weakening of the preferences. For $b=0$ full indifference is obtained, $a_{i j}^{b}=1$, while $b<0$ corresponds to preference reversal. Moreover, transformation (9) is not only reciprocity-preserving, but consistency-preserving too. That is, if $\mathbf{A}=\left[a_{i j}\right]$ is consistent, then also $\hat{\mathbf{A}}=\left[\hat{a}_{i j}\right]=\left[a_{i j}^{b}\right]$ is consistent. The proof is straightforward, since from $a_{i j} a_{j k}=a_{i k}$ immediately follows $a_{i j}^{b} a_{j k}^{b}=a_{i k}^{b}$. Uniqueness of (9) as continuous consistencypreserving transformation can be proved with a reasoning that is similar to the one described above for reciprocity.

The same function (9) has been used in 2004 by Herrera-Viedma et al. to rescale the entries of a pairwise comparison matrix from an arbitrary interval $[1 / a, a]$ into the interval $[1 / 9,9]$ (HerreraViedma et al, 2004) and by Fedrizzi and Brunelli in the ir definition of consistency equivalence classes (Fedrizzi and Brunelli, 2009). Let us finally formalize the third property as follows. For every pairwise comparison matrix $\mathbf{A}=\left[a_{i j}\right] \in A$, it must be

$$
I\left(\mathbf{A}^{b}\right) \geq I(\mathbf{A}) \quad \forall b>1,
$$

where $\mathbf{A}^{b}=\left[a_{i j}^{b}\right]$.

\subsection{Property 4. Weak monotonicity on single entries}

Let us try again to describe the meaning of the property before formalizing it. Let us consider a consistent matrix and choose one of its non-diagonal entries $a_{p q}$. If we increase or decrease its value, and modify its reciprocal $a_{q p}$ accordingly, while all the other entries remain unchanged, then the resulting matrix is not anymore consistent. For the so obtained matrix it is naturally $I(\mathbf{A})>v$, in agreement with property 1 . The idea underlying this fourth property is that the farther $a_{q p}$ is moved 
from the initial consistent value, the more inconsistent $\mathbf{A}$ becomes. This expresses a sort of weak monotonicity of the inconsistency with respect to a single comparison.

The formalization is as follows. As proved above, in order to preserve reciprocity, the only feasible change for $a_{p q}$ is $a_{p q} \rightarrow a_{p q}^{\delta}$. Therefore, given a consistent matrix $\mathbf{A} \in A^{*}$, let $\mathbf{A}(\delta)$ be the inconsistent matrix obtained from $\mathbf{A}$ by replacing the entry $a_{p q}$ with $a_{p q}^{\delta}$. Necessarily, $a_{q p}$ must be replaced with $a_{q p}^{\delta}$, while all the other entries of $\mathbf{A}$ remain unchanged. Let $\mathbf{A}\left(\delta^{\prime}\right)$ be the inconsistent matrix obtained from $\mathbf{A}$ by replacing the entry $a_{p q}$ with $a_{p q}^{\delta^{\prime}}$ and $a_{q p}$ with $a_{q p}^{\delta^{\prime}}$, while all the other entries of $\mathbf{A}$ remain unchanged. The property can then be formulated as

$$
\begin{gathered}
\delta^{\prime}>\delta>1 \quad \Rightarrow \quad I\left(\mathbf{A}\left(\delta^{\prime}\right)\right) \geq I(\mathbf{A}(\delta)) \\
\delta^{\prime}<\delta<1 \quad \Rightarrow \quad I\left(\mathbf{A}\left(\delta^{\prime}\right)\right) \geq I(\mathbf{A}(\delta)) .
\end{gathered}
$$

An equivalent definition of this property is that $I(\mathbf{A}(\delta))$ must be a non decreasing function of $\delta$ for $\delta>1$ and a non increasing function of $\delta$ for $\delta<1$. Note that property 4 involves the modification of a single comparison starting from a consistent matrix, while property 3 involves all the entries of an arbitrary matrix in $A$.

\section{Conclusions}

This paper has introduced some characterizing properties for inconsistency indices in the context of the analytic hierarchy process. We consider these properties very basic and hardly questionable, so that they should be satisfied by every index which is to be considered an inconsistency index. Future research will investigate which known inconsistency indices respect or do not respect such properties and whether the four properties are independent or dependent from each other.

\section{REFERENCES}

Aczel, J. (1966). Lectures on Functional Equations and their Applications. New York: Academic Press.

Aguarón J. and Moreno-Jimènez J. M. (2003). The geometric consistency index: Approximated threshold. European Journal of Operational Research, 147, 137-145.

Barzilai J. (1998). Consistency measures for pairwise comparison matrices. Journal of Multi-Criteria Decision Analysis, 7(3), 123-132.

Cavallo B. and D'Apuzzo L. (2009). A general unified framework for pairwise comparison matrices in multicriterial methods. International Journal of Intelligent Systems, 24, 377-398.

Cavallo B. and D'Apuzzo L. (2010). Characterizations of consistent pairwise comparison matrices over abelian linearly ordered groups. International Journal of Intelligent Systems, 25(10), 1035-1059.

Crawford G. and Williams C. (1985). A note on the analysis of subjective judgement matrices. Journal of Mathematical Psychology, 29, 25-40.

Duszak Z. and Koczkodaj W. W. (1994). Generalization of a new definition of consistency for pairwise comparisons. Information Processing Letters, 52, 273-276.

Fedrizzi M. and Brunelli M. (2009). Fair cons istency evaluation in reciprocal relations and group decision making. New Mathematics and Natural Computation, 5(2), 407-420. 
Golden B. L. and Wang Q. (1989). An alternate measure of consistency. In Golden B. L., Wasil E. A. and Harker P. T. (Eds.), The Analythic Hierarchy Process, Applications and studies (pp. 68-81).

Berlin-Heidelberg: Springer-Verlag.

Herrera-Viedma, E., Herrera, F., Chiclana, F. and Luque, M. (2004). Some issues on consistency of fuzzy preference relations. European Journal of Operational Research, 154(1), 98-109.

Koczkodaj W. W. (1993). A new definition of consistency of pairwise comparisons. Mathematical \& Computer Modelling 18(7), 79-84.

Lamata M. T. and Pelàez J.I. (2002). A method for improving the consistency of judgments. International Journal of Uncertainty, Fuzziness and Knowledge-Based Systems, 10(6) 677-686.

Obata, T., Shiraishi, S., Daigo, M. and Nakajima, N. (1999). Assessment for an incomplete matrix and improvement of the inconsistent comparison: computational experiments. Proceedings of the fifth ISAHP, 1999, Kobe, Japan, 200-205.

Peláez J.I. and Lamata M.T. (2003). A new measure of inconsistency for positive reciprocal matrices. Computer and Mathematics with Applications, 46, 1839-1845.

Ramik J. and Korviny P. (2010). Inconsistency of pair-wise comparison matrix with fuzzy elements based on geometric mean. Fuzzy Sets and Systems, 161(11), 1604-1613.

Ramik J. and Perzina R. (2010). A method for solving fuzzy multicriteria decision problems with dependent criteria. Fuzzy Optimization and Decision Making, 9(2), 123-141.

Saaty, T.L. (1977). A scaling method for priorities in hierarchical structures. Journal of Mathematical Psychology, 15, 234-281.

Shiraishi, S., Obata, T., Daigo, M. (1998). Properties of a positive reciprocal matrix and the ir application to AHP. Journal of the Operations Research Society of Japan, 41, 404-414.

Stein W. E. and Mizzi P. J. (2007). The harmonic consistency index for the analytic hierarchy process. European Journal of Operational Research, 177, 488-497.

Xu Z. and Cuiping W. (1999). A consistency improving method in the analytic hierarchy process. European Journal of Operational Research, 116(2), 443-449. 